\title{
A New Unconditionally Stable Method for Telegraph Equation Based on Associated Hermite Orthogonal Functions
}

\author{
Di Zhang, Fusheng Peng, and Xiaoping Miao \\ College of Defense Engineering, PLA University of Science and Technology, Nanjing 210007, China \\ Correspondence should be addressed to Xiaoping Miao; mxp57nj@163.com
}

Received 22 September 2016; Accepted 4 December 2016

Academic Editor: Stephen C. Anco

Copyright (C) 2016 Di Zhang et al. This is an open access article distributed under the Creative Commons Attribution License, which permits unrestricted use, distribution, and reproduction in any medium, provided the original work is properly cited.

\begin{abstract}
The present paper proposes a new unconditionally stable method to solve telegraph equation by using associated Hermite (AH) orthogonal functions. Unlike other numerical approaches, the time variables in the given equation can be handled analytically by AH basis functions. By using the Galerkin's method, one can eliminate the time variables from calculations, which results in a series of implicit equations. And the coefficients of results for all orders can then be obtained by the expanded equations and the numerical results can be reconstructed during the computing process. The precision and stability of the proposed method are proved by some examples, which show the numerical solution acquired is acceptable when compared with some existing methods.
\end{abstract}

\section{Introduction}

In this work, the following $1 d$ telegraph equation is considered:

$$
\begin{aligned}
\frac{\partial^{2} u}{\partial t^{2}}+2 \alpha \frac{\partial u}{\partial t}+\beta^{2} u=\frac{\partial^{2} u}{\partial x^{2}}+f(x, t), & \\
& a \leq x \leq b, t \geq 0
\end{aligned}
$$

with the initial conditions

$$
\begin{aligned}
u(x, 0) & =h_{0}(x), \\
\frac{\partial u(x, 0)}{\partial t} & =h_{1}(x),
\end{aligned}
$$

$$
a \leq x \leq b
$$

and the Dirichlet boundary conditions

$$
\begin{aligned}
& u(a, t)=g_{0}(t), \\
& u(b, t)=g_{1}(t), \\
& t \geq 0,
\end{aligned}
$$

where $\alpha>0$ and $\beta>0$ are real constants. We now assume that $g_{0}(t), g_{1}(t)$ and $h_{0}(x), h_{1}(x)$ are continuous functions of $t$ and $x$, respectively. The telegraph equation has been arisen in the propagation of electrical signals in wave phenomena and transmission line.

During past years, much literatures have paid attention to the analysis and development of telegraph equation, see, for example, [1-4]. Mohanty et al. [5-7] developed the finite difference method for solving telegraph equation and it has been proved to be of high accuracy. Dehghan and Shokri [8] proposed a new scheme for $1 d$ telegraph equation using collocation points and radial basis function. In [2], a highorder accurate scheme is introduced for telegraph equation. Dehghan and Ghesmati [9] developed the boundary integral equation scheme for solving second-order hyperbolic equation. Saadatmandi and Dehghan [10] proposed the Chebyshev Tau method for telegraph equation. In [11], the Chebyshev cardinal function is used for solving the $1 d$ telegraph equation. L.-B. Liu and H.-W. Liu [12] proposed a numerical solution for telegraph equation by applying the trapezoidal formula and quartic spline. Authors of [13-16] developed the radial basis function scheme for solving the telegraph equation. In recent years, some numerical schemes were proposed to solve (1) based on B-spline collocation method [17-19], polynomial scaling functions [20, 21], shifted Gegenbauer pseudospectral method [22], and differential 
quadrature algorithm [23]. These schemes are conditionally stable.

To construct an unconditional stability method to solve telegraph equation, Mohanty et al. [24-26] developed threelevel alternating direction implicit scheme to solve telegraph equation. Borhanifar and Abazari [27] proposed the parallel difference method, which is unconditionally stable, to solve telegraph equation. In [28], Mohanty presented a new unconditionally stable method to solve telegraph equation, where two parameters were introduced. Gao and Chi [29] presented two semidiscretion schemes for solving the hyperbolic equations. H.-W. Liu and L.-B. Liu [30] developed the spline difference method to solve $1 d$ problem. In [31], the compact difference scheme is proposed for solving the telegraph equation. Xie et al. [32] developed a compact difference and ADI scheme for telegraph equations with a fourth order in space. Some high-order methods, using the Padé approximation method [33] and cubic Hermite interpolation [34], were proposed for the telegraph equations.

In this study, we attempt to solve the telegraph equation using associated Hermite (AH) orthogonal functions. The Hermite functions, which were widely studied in the Hermite spectral method (HSM), are constructed by Hermite basis functions with a translated and scaled weighting function [35]. Kavian and Funaro [36] considered the use of HSM combined with variable transformation technique to solve the diffusion problems in unbounded domains. In [37, 38], Guo et al. developed the Hermite spectral method and Hermite pseudospectral methods (HPSM), and Alıc1 [39] used the HPSM to solve the $2 d$ Schrödinger equation. To stabilize the methods, a time-dependent parameter [40-42] is introduced for traditional Hermite functions to construct a conditional stability method. Unfortunately, the Hermite orthogonal functions were not used to structure an unconditional stability method for telegraph equation.

The fundamental aim of the literature is eliminating of the time variables in telegraph equation and then construct a new unconditionally stable method, the accuracy of which is absolutely independent of temporal step. In our presented method, the time variables in (1) are handled by the AH basis functions at the first step. Then, the Galerkin's method and the central difference method are introduced to this handled equation; a series of equations without time variables can be obtained. Finally, we can solve this telegraph equation by using the chasing method and the numerical solution could be reconstructed by using coefficients of $\mathrm{AH}$ functions.

\section{Associated Hermite Functions}

AH functions can be expressed as

$$
\left\{\phi_{p}(t)=\left(2^{p} p ! \pi^{1 / 2}\right)^{-1 / 2} e^{-t^{2} / 2} H_{p}(t)\right\}
$$

where the Hermite polynomials $H_{p}(t)$ is

$$
H_{p}(t)=(-1)^{p} e^{t^{2}}\left(\frac{d^{p}}{d t^{p}}\right)\left(e^{-t^{2}}\right) .
$$

The Hermite polynomials satisfy the following recursive relationship [43]:

$$
\begin{aligned}
H_{0}(t) & =1, \\
H_{1}(t) & =2 t \\
H_{p+1}(t) & =2 t H_{p}(t)-2 p H_{p-1}(t) .
\end{aligned}
$$

Then we have the derivative relations of Hermite polynomials:

$$
\begin{aligned}
& H_{p}^{\prime}(t)=2 p H_{p-1}(t), \quad p \geq 1 \\
& H_{p}^{\prime}(t)=2 t H_{p}(t)-H_{p+1}(t), \quad p \geq 0 .
\end{aligned}
$$

By introducing a time-translating parameter, $\mathrm{AH}$ functions could be transformed to a causal form:

$$
\left\{\bar{\phi}_{p}(\widetilde{t})=\left(2^{p} p ! \sigma \pi^{1 / 2}\right)^{-1 / 2} e^{-\widetilde{t} / 2} H_{p}(\widetilde{t})\right\},
$$

where $\tilde{t}=\left(t-T_{f}\right) / \sigma ; T_{f}$ and $\sigma$ are the translating parameter and time-scaling parameter, respectively. A function with causal relationships can be expanded by choosing a suitable $T_{f}$ and $\sigma$ :

$$
u(x, t)=\sum_{p=0}^{\infty} u^{p}(x) \phi_{p}(\widetilde{t}) .
$$

Combining (6) and (8), we have

$$
\begin{aligned}
\bar{\phi}_{0}(\tilde{t}) & =\frac{1}{\sqrt{2 \sigma \sqrt{\pi}}} e^{-t^{2} / 2}, \\
\bar{\phi}_{1}(\tilde{t}) & =\frac{1}{\sqrt{2 \sigma \sqrt{\pi}}} \sqrt{2} t e^{-t^{2} / 2}, \\
\bar{\phi}_{p+1}(\tilde{t}) & =t \sqrt{\frac{2}{p+1}} \bar{\phi}_{p}(\tilde{t})-\sqrt{\frac{p}{p+1}} \bar{\phi}_{p-1}(\tilde{t}),
\end{aligned}
$$

$p \geq 1$

Property (7) and the above formula lead to the derivation of $\bar{\phi}_{p}(\widetilde{t})$ :

$$
\begin{aligned}
& \frac{d}{d t} \bar{\phi}_{p}(\widetilde{t}) \\
& \quad \begin{cases}-\frac{1}{\sigma} \sqrt{\frac{1}{2}} \bar{\phi}_{1}(\widetilde{t}), & (p=0), \\
\frac{1}{\sigma} \sqrt{\frac{p}{2}} \bar{\phi}_{p-1}(\tilde{t})-\frac{1}{\sigma} \sqrt{\frac{p+1}{2}} \bar{\phi}_{p+1}(\tilde{t}), & (p \geq 1) .\end{cases}
\end{aligned}
$$


Advances in Mathematical Physics

3

Combining (9) and (11), we have

$$
\begin{aligned}
& \frac{\partial u(x, t)}{\partial t}=\sum_{p=0}^{\infty} u^{p}(x) \frac{d}{d t} \bar{\phi}_{p}(\tilde{t}) \\
& =u^{0}(x) \frac{d}{d t} \bar{\phi}_{0}(\tilde{t})+\sum_{p=1}^{\infty} u^{p}(x) \frac{d}{d t} \bar{\phi}_{p}(\tilde{t}) \\
& =u^{0}(x)\left[-\sqrt{\frac{1}{2 \sigma^{2}}} \bar{\phi}_{1}(\tilde{t})\right] \\
& \quad+\sum_{p=1}^{\infty} u^{p}(x)\left[\sqrt{\frac{p}{2 \sigma^{2}}} \bar{\phi}_{p-1}(\tilde{t})-\sqrt{\frac{p+1}{2 \sigma^{2}}} \bar{\phi}_{p+1}(\tilde{t})\right] .
\end{aligned}
$$

Let $m=p-1$; we can obtain

$$
\begin{aligned}
& \frac{\partial u(x, t)}{\partial t}=u^{0}(x)\left[-\sqrt{\frac{1}{2 \sigma^{2}}} \bar{\phi}_{1}(\tilde{t})\right]+\sum_{m=0}^{\infty} u^{m+1}(x) \\
& \cdot\left[\sqrt{\frac{m+1}{2 \sigma^{2}}} \bar{\phi}_{m}(\widetilde{t})-\sqrt{\frac{m+2}{2 \sigma^{2}}} \bar{\phi}_{m+2}(\tilde{t})\right] \\
& =\sum_{m=0}^{\infty} u^{m+1}(x) \sqrt{\frac{m+1}{2 \sigma^{2}}} \bar{\phi}_{m}(\tilde{t})+u^{0}(x) \\
& \cdot\left[-\sqrt{\frac{1}{2 \sigma^{2}} \bar{\phi}_{1}}(\tilde{t})\right]+\sum_{m=0}^{\infty} u^{m+1}(x) \\
& \cdot\left[-\sqrt{\frac{m+2}{2 \sigma^{2}}} \bar{\phi}_{m+2}(\tilde{t})\right] .
\end{aligned}
$$

Let $u^{-1}(x)=0$; we have

$$
\begin{aligned}
& u^{0}(x)\left[-\sqrt{\frac{1}{2 \sigma^{2}}} \bar{\phi}_{1}(\tilde{t})\right] \\
& +\sum_{m=0}^{\infty} u^{m+1}(x)\left[-\sqrt{\frac{m+2}{2 \sigma^{2}}} \bar{\phi}_{m+2}(\tilde{t})\right] \\
& \quad \stackrel{k=m+2}{=} u^{0}(x)\left[-\sqrt{\frac{1}{2 \sigma^{2}}} \bar{\phi}_{1}(\tilde{t})\right] \\
& +\sum_{k=2}^{\infty} u^{k-1}(x)\left[-\sqrt{\frac{k}{2 \sigma^{2}}} \bar{\phi}_{k}(\tilde{t})\right] \\
& =\sum_{k=0}^{\infty} u^{k-1}(x)\left[-\sqrt{\frac{k}{2 \sigma^{2}}} \bar{\phi}_{k}(\tilde{t})\right] .
\end{aligned}
$$




$$
\begin{array}{r}
=\sum_{k=0}^{\infty}\left[u^{k}(x) \sqrt{\frac{k}{2 \sigma^{2}}}-u^{k-2}(x) \sqrt{\frac{k-1}{2 \sigma^{2}}}\right] \\
\cdot\left[-\sqrt{\frac{k}{2 \sigma^{2}}} \bar{\phi}_{k}(\tilde{t})\right] \\
\text { Let }\left(u^{-2}(x)=0, u^{-1}(x)=0\right) .
\end{array}
$$

From the above equations, we can obtain the first and the second partial derivative of $u(x, t)$ :

$$
\begin{aligned}
& \frac{\partial u(x, t)}{\partial t}=\sum_{p=0}^{\infty}\left[u^{p+1}(x) \sqrt{\frac{p+1}{2 \sigma^{2}}}-u^{p-1}(x) \sqrt{\frac{p}{2 \sigma^{2}}}\right] \\
& \cdot \bar{\phi}_{p}(\tilde{t}) \\
& \frac{\partial^{2} u(x, t)}{\partial t^{2}}=\sum_{m=0}^{\infty}\left[u^{m+2}(x) \sqrt{\frac{m+2}{2 \sigma^{2}}}\right. \\
& \left.-u^{m}(x) \sqrt{\frac{m+1}{2 \sigma^{2}}}\right] \cdot \sqrt{\frac{m+1}{2 \sigma^{2}}} \bar{\phi}_{m}(\tilde{t}) \\
& +\sum_{k=0}^{\infty}\left[u^{k}(x) \sqrt{\frac{k}{2 \sigma^{2}}}-u^{k-2}(x) \sqrt{\frac{k-1}{2 \sigma^{2}}}\right] \\
& {\left[-\sqrt{\frac{k}{2 \sigma^{2}}} \bar{\phi}_{k}(\tilde{t})\right]} \\
& \stackrel{m=p, k=p}{=} \sum_{p=0}^{\infty}\left[u^{p+2}(x) \sqrt{\frac{p+2}{2 \sigma^{2}}} \sqrt{\frac{p+1}{2 \sigma^{2}}}\right. \\
& \left.-u^{p}(x) \frac{2 p+1}{2 \sigma^{2}}+u^{p-2}(x) \sqrt{\frac{p-1}{2 \sigma^{2}}} \sqrt{\frac{p}{2 \sigma^{2}}}\right] \cdot \bar{\phi}_{p}(\tilde{t}) .
\end{aligned}
$$

\section{Description of the Method}

3.1. Construction of Computing Matrix. The partial differential with respect to $x$ can be written as

$$
\frac{\partial^{2} u}{\partial x^{2}}=\sum_{p=0}^{\infty} \frac{\partial^{2}}{\partial x^{2}} u^{p}(x) \bar{\phi}_{p}(\widetilde{t})
$$

Rewriting the telegraph equation (1) using (9), (18), and (19), we can obtain

$$
\begin{aligned}
& \sum_{p=0}^{\infty}\left[u^{p+2}(x) \sqrt{\frac{p+2}{2 \sigma^{2}}} \sqrt{\frac{p+1}{2 \sigma^{2}}}-u^{p}(x) \frac{2 p+1}{2 \sigma^{2}}\right. \\
& \left.+u^{p-2}(x) \sqrt{\frac{p-1}{2 \sigma^{2}}} \sqrt{\frac{p}{2 \sigma^{2}}}\right] \cdot \bar{\phi}_{p}(\tilde{t})
\end{aligned}
$$

$$
\begin{aligned}
& +2 \alpha \sum_{p=0}^{\infty}\left[u^{p+1}(x) \sqrt{\frac{p+1}{2 \sigma^{2}}}-u^{p-1}(x) \sqrt{\frac{p}{2 \sigma^{2}}}\right] \\
& \cdot \bar{\phi}_{p}(\tilde{t})+\beta^{2} \sum_{p=0}^{\infty} u^{p}(x) \phi_{p}(\tilde{t})=\sum_{p=0}^{\infty} \frac{\partial^{2}}{\partial x^{2}} u^{p}(x) \bar{\phi}_{p}(\tilde{t}) \\
& +\sum_{p=0}^{\infty} f^{p}(x) \bar{\phi}_{p}(\tilde{t}) .
\end{aligned}
$$

In (20), we introduced a Galerkin procedure [44] in order to eliminate the time variable terms $\bar{\phi}_{p}(\widetilde{t})$. By multiplying each side of (20) by time-dependent term $\bar{\phi}_{q}(\tilde{t})$, we can obtain the following equation by integrating over time from 0 to $\infty$ :

$$
\begin{aligned}
& \sqrt{\frac{q+1}{2}} \sqrt{\frac{q+2}{2}} u^{q+2}(x)-\frac{2 q+1}{2} u^{q}(x) \\
& +\sqrt{\frac{q-1}{2}} \sqrt{\frac{q}{2}} u^{q-2}(x) \\
& +2 \alpha \sigma\left[\sqrt{\frac{q+1}{2}} u^{q+1}(x)-\sqrt{\frac{q}{2}} u^{q-1}(x)\right] \\
& +\sigma^{2} \beta^{2} u^{q}(x)=\sigma^{2} \frac{\partial^{2}}{\partial x^{2}} u^{q}(x)+\sigma^{2} f^{q}(x),
\end{aligned}
$$

where

$$
f^{q}(x)=\int_{-T_{f} / 2}^{T_{f} / 2} f(x, t) \bar{\phi}_{q}(\widetilde{t}) d t .
$$

Discretize (21) using the central difference scheme in space; we have

$$
\begin{aligned}
& \left.\sqrt{\frac{q+1}{2}} \sqrt{\frac{q+2}{2}} u^{q+2}(x)\right|_{i}-\left.\frac{2 q+1}{2} u^{q}(x)\right|_{i} \\
& +\left.\sqrt{\frac{q-1}{2}} \sqrt{\frac{q}{2}} u^{q-2}(x)\right|_{i} \\
& \quad+2 \alpha \sigma\left[\left.\sqrt{\frac{q+1}{2}} u^{q+1}(x)\right|_{i}-\left.\sqrt{\frac{q}{2}} u^{q-1}(x)\right|_{i}\right] \\
& \quad+\left.\sigma^{2} \beta^{2} u^{q}(x)\right|_{i} \\
& =\frac{\sigma^{2}}{\Delta x_{i}^{2}}\left(\left.u^{q}\right|_{i+1}-\left.2 u^{q}\right|_{i}+\left.u^{q}\right|_{i-1}\right)+\left.\sigma^{2} f^{q}(x)\right|_{i} .
\end{aligned}
$$

From (23), one could conclude that the variable of the $q$ order is connected with adjacent fields, from $q-2$ to $q+2$. In (23), $\left.u^{q}(x)\right|_{i}$ is moved to the left side of the equation and simplified to

$$
[\eta][u]_{i}=[\lambda] \frac{\sigma^{2}}{\Delta x_{i}^{2}}\left([u]_{i+1}+[u]_{i-1}\right)+\sigma^{2}[f]_{i},
$$

where 


$$
[\eta]=\left[\begin{array}{cccc}
\frac{2 \sigma^{2}}{\Delta x_{i}^{2}}+\sigma^{2} \beta^{2}-\frac{1}{2} & 2 \alpha \sigma \sqrt{\frac{1}{2}} & \sqrt{\frac{1}{2}} \sqrt{\frac{2}{2}} & \\
-2 \alpha \sigma \sqrt{\frac{1}{2}} & \frac{2 \sigma^{2}}{\Delta x_{i}^{2}}+\sigma^{2} \beta^{2}-\frac{3}{2} & 2 \alpha \sigma \sqrt{\frac{2}{2}} & \sqrt{\frac{2}{2} \sqrt{\frac{3}{2}}} \\
\ddots & \ddots & \ddots & 2 \alpha \sigma \sqrt{\frac{Q-1}{2}} \\
\sqrt{\frac{Q-3}{2} \sqrt{\frac{Q-2}{2}}} & -2 \alpha \sigma \sqrt{\frac{Q-2}{2}} & \frac{2 \sigma^{2}}{\Delta x_{i}^{2}}+\sigma^{2} \beta^{2}-\frac{2 Q-3}{2} & 2 Q-1 \\
& \sqrt{\frac{Q-2}{2}} \sqrt{\frac{Q-1}{2}} & -2 \alpha \sigma \sqrt{\frac{Q-1}{2}} & \frac{2 \sigma^{2}}{\Delta x_{i}^{2}}+\sigma^{2} \beta^{2}-\frac{2 Q-1}{2}
\end{array}\right]_{Q \times Q}
$$

In (24), $[\lambda]=I_{\mathrm{Q} \times \mathrm{Q}}$ is an unit matrix, and $[u]_{i}=\left[\left.u\right|_{i} ^{0}, \cdots\right.$, $\left.\left.u\right|_{i} ^{\mathrm{Q}-2},\left.u\right|_{i} ^{\mathrm{Q}-1}\right]_{1 \times \mathrm{Q}}^{T}$ is a Q-tupple matrix of $\mathrm{AH}$ space for numerical results.

Rewriting (24) with a nested matrix, we have

$$
[A]\{[u]\}=\{[F]\} \quad q=0,1,2, \ldots,
$$

where

$$
=\left[\begin{array}{cccccc}
{[\eta]} & -\frac{\sigma^{2}}{\Delta x_{i}^{2}}[\lambda] & & & \\
-\frac{\sigma^{2}}{\Delta x_{i}^{2}}[\lambda] & {[\eta]} & -\frac{\sigma^{2}}{\Delta x_{i}^{2}}[\lambda] & & \\
& -\frac{\sigma^{2}}{\Delta x_{i}^{2}}[\lambda] & {[\eta]} & -\frac{\sigma^{2}}{\Delta x_{i}^{2}}[\lambda] & \\
& & \ddots & \ddots & \ddots \\
& & & -\frac{\sigma^{2}}{\Delta x_{i}^{2}}[\lambda] & {[\eta]}
\end{array}\right] .
$$

In (26), $\{[u]\}$ is a combination of numerical result with all orders and all points. $\{[F]\}$ represents source coefficients in the computational domain. $[A]$ is a banded sparse matrix with $Q$ submatrix elements.

3.2. Method's Treatment of Boundary and Initial Conditions. The application of presented method to telegraph equation based on a concealment condition that the initial valve of the equation should be 0 ; that is $h_{0}(x)=h_{1}(x)=0$. For the nonhomogeneous initial condition, we have

$$
w(x, t)=u(x, t)-h_{0}(x)-t h_{1}(x) .
$$

For the Dirichlet boundary conditions, there are a couple of ways to deal with it. The point on the boundary would be regarded as a point source which changes over time in the first approach. Then we can expand $w(a, t), w(b, t)$, and $f(x, t)$ using $\mathrm{AH}$ basis functions, respectively. However, this kind of method has the drawback of complicated procedure and a little loss in accuracy. In this work, the second approach has been adopted in which the Dirichlet boundary conditions are homogenized. We apply

$$
v(x, t)=w(x, t)-\left[w(a, t)+\frac{w(b, t)-w(a, t)}{b-a} x\right] .
$$

Introducing (28) and (29) to (1)-(3), we can obtain a telegraph equation with the homogeneous boundary and initial conditions

$$
\begin{array}{ll}
v_{t t}(x, t)+2 \alpha v_{t}(x, t)+\beta^{2} v(x, t) & \\
=v_{x x}(x, t)+M(x, t), \quad a \leq x \leq b, t \geq 0, & \\
v(x, 0)=0, & a \leq x \leq b, \\
v_{t}(x, 0)=0, & \\
v(a, t)=0, & \\
v(b, t)=0, & t \geq 0 .
\end{array}
$$

Rewriting (22) and (26) we have

$$
\begin{gathered}
M^{q}(x)=\int_{-T_{f} / 2}^{T_{f} / 2} M(x, t) \bar{\phi}_{q}(\widetilde{t}) d t, \\
{[A]\{[v]\}=\{[M]\} \quad q=0,1,2, \ldots}
\end{gathered}
$$

To solve (32), the lower-upper (LU) decomposition scheme is applied to decompose $[A]$ initially, and iteration method is used to obtain the numerical results. The LU decomposition of $[A]$ is handled only once when starting calculation process. Finally, one can obtain the numerical result from the expansion coefficients as

$$
u(x, t)=\sum_{q=0}^{Q-1} u^{q}(x) \bar{\phi}_{q}(\widetilde{t}) .
$$

Contrary to the other numerical approach, this presented method has an implicit relationship in each variables, which can be reflected in the sparse matrix $[A]$. One should notice that (32) is independent of the temporal testing procedure $\bar{\phi}_{p}(\tilde{t})$. And the matrix $[A]$, which does not contain the order $q$, remains unchanged in the calculation process. Therefore, the temporal step does not affect the stability of this method any more, and then an unconditionally stable scheme is structured. In this presented method, the time step size is only 
TABLE 1: Comparison of RMS error in Example 1 with $\lambda=1.6$ at $t=2 \mathrm{~s}$.

\begin{tabular}{|c|c|c|c|c|c|}
\hline Methods & $h$ & $\alpha=10, \beta=3$ & $\alpha=100, \beta=3$ & $\alpha=10, \beta=5$ & $\alpha=100, \beta=5$ \\
\hline \multirow{3}{*}{ Method in [28] } & $1 / 8$ & $0.7316(-03)$ & $0.1094(-02)$ & $0.2252(-02)$ & $0.2636(-02)$ \\
\hline & $1 / 16$ & $0.1826(-04)$ & $0.6757(-04)$ & $0.8022(-04)$ & $0.1708(-03)$ \\
\hline & $1 / 32$ & $0.3995(-05)$ & $0.2023(-05)$ & $0.3351(-05)$ & $0.8244(-05)$ \\
\hline \multirow{3}{*}{ Method in [30] } & $1 / 8$ & $0.2528(-03)$ & $0.5542(-02)$ & $0.1196(-02)$ & $0.3135(-02)$ \\
\hline & $1 / 16$ & $0.3620(-04)$ & $0.4652(-04)$ & $0.9672(-04)$ & $0.8555(-03)$ \\
\hline & $1 / 32$ & $0.5196(-04)$ & $0.2305(-04)$ & $0.3528(-05)$ & $0.3215(-04)$ \\
\hline \multirow{3}{*}{ Method in [12] } & $1 / 8$ & $0.3044(-03)$ & $0.5616(-03)$ & $0.2079(-03)$ & $0.5047(-03)$ \\
\hline & $1 / 16$ & $0.3792(-04)$ & $0.7000(-04)$ & $0.2535(-04)$ & $0.6291(-04)$ \\
\hline & $1 / 32$ & $0.4709(-05)$ & $0.8741(-05)$ & $0.3109(-05)$ & $0.7854(-05)$ \\
\hline \multirow{3}{*}{ Proposed method } & $1 / 8$ & $8.3635(-06)$ & $1.2673(-04)$ & $6.9791(-05)$ & $6.3081(-05)$ \\
\hline & $1 / 16$ & $3.5515(-06)$ & $2.0919(-05)$ & $8.1144(-06)$ & $8.3177(-06)$ \\
\hline & $1 / 32$ & $8.3032(-07)$ & $1.1860(-06)$ & $8.4148(-07)$ & $4.4470(-06)$ \\
\hline
\end{tabular}

applied to compute the $\mathrm{AH}$ coefficients of the source term due to (22) and (31), which has been done at the beginning of the computation. We can choose a relatively small value of time step to calculate (22) or (31) accurately and describe the process of propagation of electrical signals clearly, and this procedure does not increase the computation time.

\section{Numerical Examples}

In this part, the following four examples of the telegraph equation with exact solution have been solved by the proposed scheme. To measure the accuracy and versatility of this proposed scheme, the following $L_{2}, L_{\infty}$, and root-meansquare (RMS) errors are reported:

$$
\begin{aligned}
L_{2} & =\left\|u^{\text {exact }}-u^{\text {num }}\right\|_{2}=\sqrt{h \sum_{j=0}^{N}\left|u_{j}^{\text {exact }}-u_{j}^{\text {num }}\right|^{2}}, \\
L_{\infty} & =\left\|u^{\text {exact }}-u^{\text {num }}\right\|_{\infty}=\max _{j}\left|u_{j}^{\text {exact }}-u_{j}^{\text {num }}\right|, \\
\text { RMS } & =\sqrt{\frac{\sum_{j=0}^{N}\left|u_{j}^{\text {exact }}-u_{j}^{\text {num }}\right|^{2}}{N+1}} .
\end{aligned}
$$

Example 1. We consider the telegraph equation:

$$
\begin{gathered}
u_{t t}(x, t)+2 \alpha u_{t}(x, t)+\beta^{2} u(x, t) \\
=u_{x x}(x, t)+\left(\beta^{2}-2\right) \sinh x \cos t \\
-2 \alpha \sinh x \sin t,
\end{gathered}
$$

over a region $\Omega=[0 \leq x \leq 1] \times[t>0]$, with initial conditions

$$
\begin{aligned}
& u(x, 0)=\sinh x, \\
& u_{t}(x, 0)=0
\end{aligned}
$$

and boundary conditions

$$
\begin{aligned}
& u(0, t)=0 \\
& u(1, t)=\sinh 1 \cos t
\end{aligned}
$$

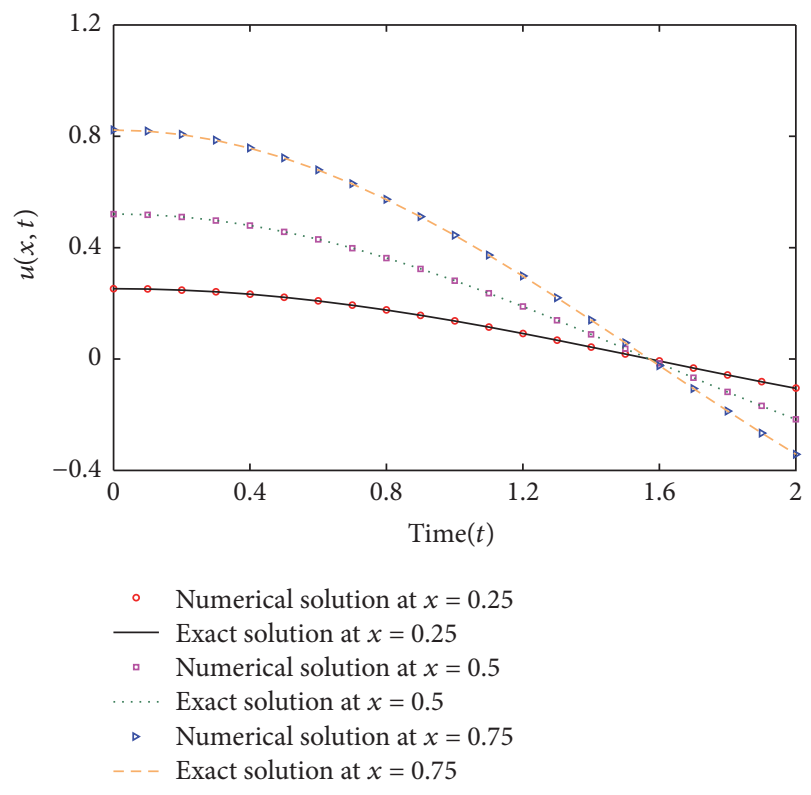

FIGURE 1: Comparison of analytical and numerical result of Example 1 at different space level with $h=0.01, k=0.1$.

The exact solution of this equation is $u(x, t)=$ $\sinh x \cos t$. The results for different value of $\alpha$ and $\beta$ obtained by using the proposed method are compared with those obtained by Mohanty [28], H.-W. Liu and L.-B. Liu [30] and L.-B. Liu and H.-W. Liu [12], which are presented in Tables 1 and 2 . The graph of analytical and numerical result at $x=$ $0.25, x=0.5$ and $x=0.75$ is shown in Figure 1 .

Example 2. In this case, the telegraph equation (1) with $\alpha=$ $4, \beta=2$ over a region $[0, \pi]$ with the following boundary and initial conditions was considered:

$$
\begin{aligned}
& u(x, 0)=\sin x, \\
& u_{t}(x, 0)=-\sin x, \\
& \quad 0 \leq x \leq \pi, \\
& u(0, t)=u(\pi, t)=0, \quad t \geq 0 .
\end{aligned}
$$


TABLE 2: Comparison of RMS error in Example 1 with $\lambda=3.2$ at $t=2 \mathrm{~s}$.

\begin{tabular}{|c|c|c|c|c|c|}
\hline Methods & $h$ & $\alpha=10, \beta=2$ & $\alpha=100, \beta=2$ & $\alpha=10, \beta=5$ & $\alpha=100, \beta=5$ \\
\hline \multirow{3}{*}{ Method in [28] } & $1 / 8$ & $0.4116(-02)$ & $0.6115(-02)$ & $0.1149(-01)$ & $0.1737(-01)$ \\
\hline & $1 / 16$ & $0.7773(-04)$ & $0.8872(-03)$ & $0.2174(-02)$ & $0.2570(-02)$ \\
\hline & $1 / 32$ & $0.2591(-04)$ & $0.5430(-04)$ & $0.7906(-04)$ & $0.1684(-03)$ \\
\hline \multirow{3}{*}{ Method in [30] } & $1 / 8$ & $0.5803(-02)$ & $0.2435(-02)$ & $0.2568(-02)$ & $0.1258(-01)$ \\
\hline & $1 / 16$ & $0.1306(-03)$ & $0.5218(-03)$ & $0.2294(-02)$ & $0.3035(-02)$ \\
\hline & $1 / 32$ & $0.5256(-04)$ & $0.4042(-04)$ & $0.6335(-04)$ & $0.2087(-03)$ \\
\hline \multirow{3}{*}{ Method in [12] } & $1 / 8$ & $0.1557(-02)$ & $0.2330(-02)$ & $0.8488(-03)$ & $0.2022(-02)$ \\
\hline & $1 / 16$ & $0.1952(-03)$ & $0.2900(-03)$ & $0.1040(-03)$ & $0.2519(-03)$ \\
\hline & $1 / 32$ & $0.2431(-04)$ & $0.3621(-04)$ & $0.1268(-04)$ & $0.3144(-04)$ \\
\hline \multirow{3}{*}{ Proposed method } & $1 / 8$ & $7.8006(-05)$ & $6.6504(-04)$ & $2.8559(-04)$ & $4.6424(-04)$ \\
\hline & $1 / 16$ & $1.5861(-05)$ & $1.4478(-04)$ & $1.5184(-05)$ & $7.7917(-05)$ \\
\hline & $1 / 32$ & $3.1948(-06)$ & $5.0823(-06)$ & $1.7082(-06)$ & $2.9197(-05)$ \\
\hline
\end{tabular}

TABLE 3: Errors in numerical result of Example 2 with $h=0.02, k=0.0001$ at $t=0.5$ and $t=1$.

\begin{tabular}{|c|c|c|c|c|c|c|}
\hline \multirow{2}{*}{ Methods } & \multicolumn{3}{|c|}{$t=0.5$} & \multicolumn{3}{|c|}{$t=1.0$} \\
\hline & $L_{2}$ & $L_{\infty}$ & RMS error & $L_{2}$ & $L_{\infty}$ & RMS error \\
\hline Method in [8] & $7.9491(-05)$ & $8.3721(-06)$ & $6.3239(-06)$ & $1.4554(-04)$ & $1.5680(-05)$ & $1.1579(-05)$ \\
\hline Method in [18] & $8.7500(-06)$ & $9.9900(-06)$ & $7.1600(-06)$ & $5.0700(-06)$ & $5.1900(-06)$ & $7.1700(-06)$ \\
\hline Method in [19] & $2.3328(-06)$ & $1.8612(-06)$ & - & $4.3667(-06)$ & $3.4839(-06)$ & - \\
\hline Proposed method & $6.7195(-07)$ & $7.5836(-08)$ & $5.3289(-08)$ & $1.0890(-06)$ & $1.2291(-07)$ & $8.6308(-08)$ \\
\hline
\end{tabular}

TABLE 4: RMS errors of Example 2 with $k=0.01$ at $t=3.0$.

\begin{tabular}{lcccc}
\hline$h$ & Linear RBF [9] & Cubic RBF [9] & Method in [31] & Proposed method \\
\hline 0.05 & $3.0100(-04)$ & $7.1250(-05)$ & $6.0410(-07)$ & $3.6559(-07)$ \\
0.02 & $7.1280(-05)$ & $1.7120(-05)$ & $6.0110(-07)$ & $1.4537(-07)$ \\
0.01 & $4.3200(-05)$ & $8.2180(-06)$ & $6.0001(-07)$ & $7.3283(-08)$ \\
\hline
\end{tabular}

We have $f(x, t)=\left(2-2 \alpha+\beta^{2}\right) e^{-t} \sin x$. And the exact solution of this example is $u(x, t)=e^{-t} \sin x$. The $L_{2}, L_{\infty}$, and RMS errors for $t=0.5$ and 1.0 are presented in Table 3 with $h=0.02$ and $k=0.0001$. The errors are compared with the numerical results acquired in Dehghan and Shokri [8], Sharifi and Rashidinia [18] and Mittal and Bhatia [19]. The RMS errors for $t=3.0$ with $k=0.01$ are shown in Table 4 . The numerical solutions by this proposed method are compared with the results obtained by [9] and another unconditionally stable method in [31]. The space-time graph of numerical result up to $t=2$ is shown in Figure 2.

Example 3. In this case, we consider telegraph equation (1) with $\alpha=10, \beta=5$ over region $[0,2]$, and the following boundary and initial conditions:

$$
\begin{aligned}
& u(x, 0)=\tan \left(\frac{x}{2}\right), \\
& u_{t}(x, 0)=\frac{1}{2}\left[1+\tan ^{2}\left(\frac{x}{2}\right)\right], \\
& \qquad 0 \leq x \leq 2, \\
& u(0, t)=\tan \left(\frac{t}{2}\right),
\end{aligned}
$$

FIGURE 2: Space-time graph of numerical result up to $t=2$, with $h=0.02$ and $k=0.01$ for Example 2 .

$$
u(2, t)=\tan \left(\frac{2+t}{2}\right)
$$


TABLE 5: Errors in numerical result of Example 3 with $h=0.001, k=0.001$ at different time levels.

\begin{tabular}{lcccr}
\hline Methods & Time & $L_{2}$ & $L_{\infty}$ & RMS error \\
\hline Method in [19] & $t=0.2$ & $2.1800(-04)$ & $3.6103(-04)$ & $1.0368(-04)$ \\
Method in [19] & $t=0.4$ & $5.6618(-04)$ & $6.8300(-05)$ & - \\
Method in [18] & $t=0.2$ & $9.9900(-06)$ & $4.2800(-05)$ & $6.0700(-06)$ \\
Method in [18] & $t=0.4$ & $7.0700(-06)$ & $2.5596(-07)$ & $5.2700(-06)$ \\
Proposed method & $t=0.2$ & $7.2429(-06)$ & $4.9275(-07)$ & $1.6187(-07)$ \\
Proposed method & $t=0.4$ & $6.2790(-06)$ & & $1.4033(-07)$ \\
\hline
\end{tabular}

TABLE 6: $L_{2}, L_{\infty}$, and RMS errors information in Example 4 with $h=0.01, k=0.001$.

\begin{tabular}{|c|c|c|c|c|c|}
\hline Methods & Time & $L_{2}$ & $L_{\infty}$ & RMS error & CPU time (s) \\
\hline Method in [8] & $t=1$ & $1.4386 \times 10^{-4}$ & $1.8479 \times 10^{-5}$ & $1.4315 \times 10^{-5}$ & 0 \\
\hline Method in [8] & $t=5$ & $7.5545 \times 10^{-5}$ & $1.0455 \times 10^{-5}$ & $7.5170 \times 10^{-6}$ & 2 \\
\hline Method in [19] & $t=1$ & $4.5526 \times 10^{-5}$ & $5.9153 \times 10^{-5}$ & - & 0.43 \\
\hline Method in [19] & $t=5$ & $3.0161 \times 10^{-6}$ & $5.2032 \times 10^{-6}$ & - & 1.46 \\
\hline Proposed method & $t=1$ & $1.7961 \times 10^{-6}$ & $3.4326 \times 10^{-7}$ & $1.7784 \times 10^{-7}$ & 0.34 \\
\hline Proposed method & $t=5$ & $1.4776 \times 10^{-6}$ & $2.2537 \times 10^{-7}$ & $1.4631 \times 10^{-7}$ & 0.35 \\
\hline
\end{tabular}

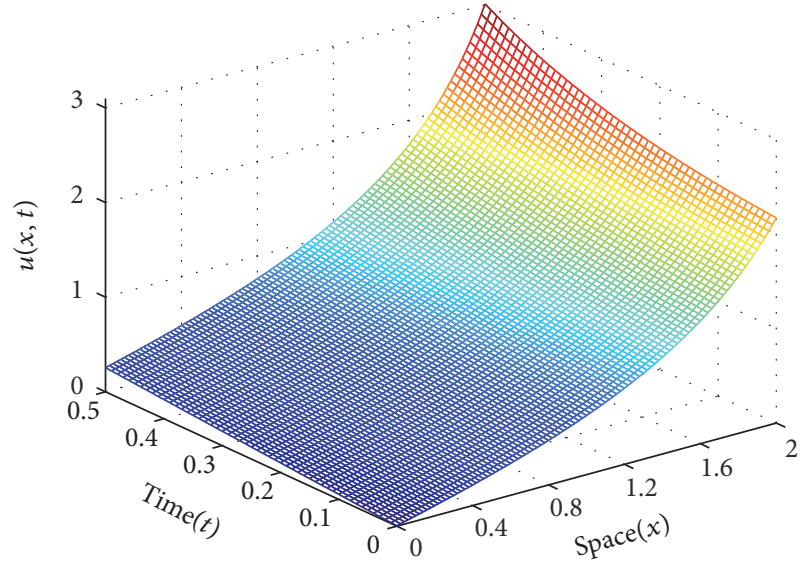

(a)

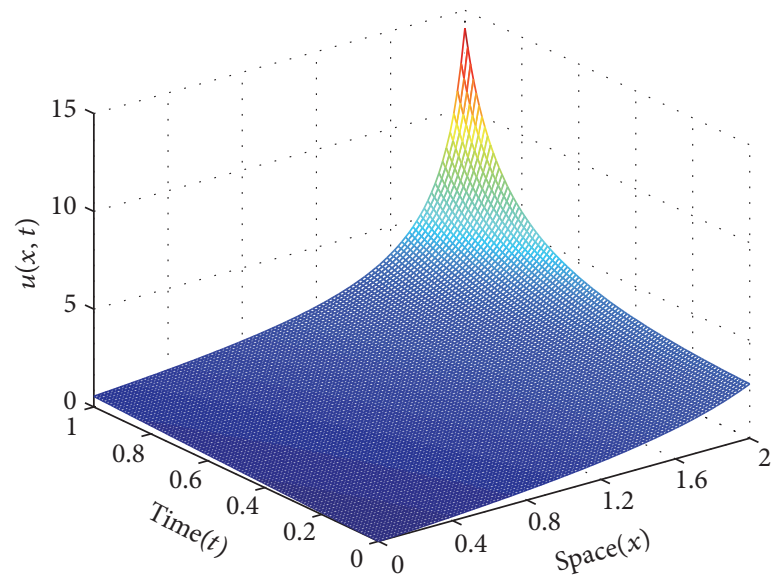

(b)

FIGURE 3: Space-time graphs of numerical solution of Example 3 with $h=0.02$ and $k=0.01$ up to $t=0.5$ (a) and $t=1$ (b).

In this problem, we have $f(x, t)=\alpha\left[1+\tan ^{2}((x+t) / 2)\right]+$ $\beta^{2} \tan ((x+t) / 2)$. And the analytical solution of the example is $f(x, t)=\tan ((x+t) / 2)$. The $L_{2}, L_{\infty}$, and RMS errors at $t=0.2$ and $t=0.4$ with $h=0.001, k=0.001$ are shown in Table 5. And also the numerical results are compared with Sharifi and Rashidinia [18] and Mittal and Bhatia [19]. The space-time graphs of numerical result with $h=0.02$ and $k=$ 0.01 up to $t=0.5$ and 1.0 are presented in Figure 3 .

Example 4. We consider telegraph equation with $\alpha=0.5$ and $\beta=1$

$$
\begin{aligned}
& u_{t t}(x, t)+u_{t}(x, t)+u(x, t) \\
& \quad=u_{x x}(x, t)+\left(2-2 t+t^{2}\right)\left(x-x^{2}\right) e^{-t}+2 t^{2} e^{-t},
\end{aligned}
$$

in the interval $0 \leq x \leq 1$, with initial conditions

$$
\begin{gathered}
u(x, 0)=0, \\
u_{t}(x, 0)=0
\end{gathered}
$$

and boundary conditions

$$
\begin{aligned}
& u(0, t)=0, \\
& u(1, t)=0 .
\end{aligned}
$$

The analytical solution of this equation is $u(x, t)=(x-$ $\left.x^{2}\right) t^{2} e^{-t}$. The CPU time and $L_{2}, L_{\infty}$, and RMS errors for $t$ $=1$ and 5 are shown in Table 6 with $h=0.01$ and $k=0.001$. The numerical solution is compared with results of $[8,19]$. It can be concluded that numerical solutions obtained by 


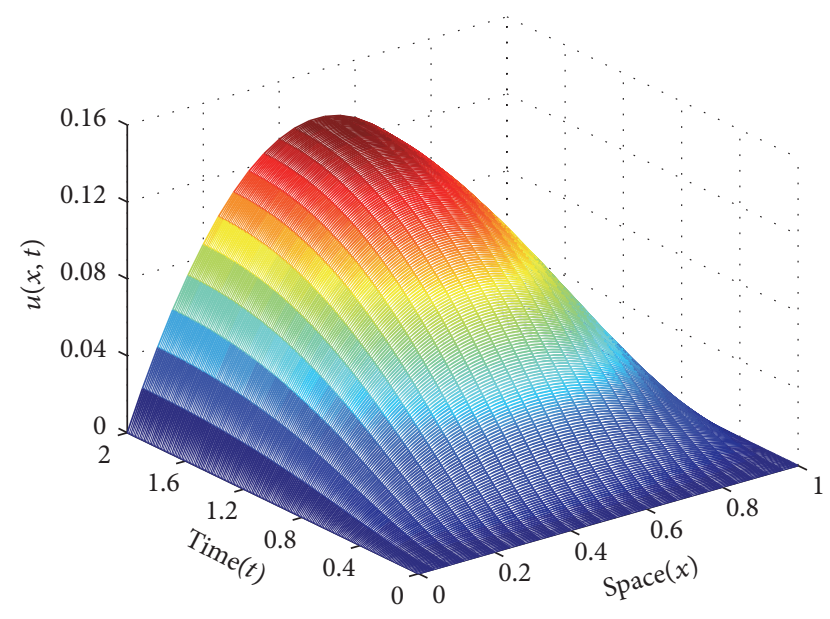

FIGURE 4: Space-time graph of numerical result up to $t=2$, with $h=$ 0.04 and $k=0.01$ for Example 4 .

this presented scheme are good in comparison with $[8,19]$. Moreover, our method has a higher efficiency than the other two methods when the computation time gets longer. The space-time graph of numerical result is shown in Figure 4.

\section{Conclusion}

In the literature, we have constructed a new unconditionally stable method to solve the telegraph equation. Since the time variables have been eliminated from computations, the convergence and precision of the presented method are independent of the temporal step. To demonstrate the stability and accuracy of this proposed method, four numerical examples are conducted and compared with the numerical results available in previous literatures. The comparison numerical results reveal the unconditional stability and high precision of the presented scheme in the current literature.

The paper has provided a new mentality for solving $1 d$ telegraph equation and this method is now extended to multidimensional cases and other kinds of partial differential equations in a future study.

\section{Competing Interests}

The authors declare that they have no competing interests regarding the publication of this paper.

\section{References}

[1] E. H. Twizell, "An explicit difference method for the wave equation with extended stability range," BIT Numerical Mathematics, vol. 19, no. 3, pp. 378-383, 1979.

[2] A. Mohebbi and M. Dehghan, "High order compact solution of the one-space-dimensional linear hyperbolic equation," Numerical Methods for Partial Differential Equations, vol. 24, no. 5, pp. 1222-1235, 2008.

[3] M. Ciment and S. H. Leventhal, "A note on the operator compact implicit method for the wave equation," Mathematics of Computation, vol. 32, no. 141, pp. 143-147, 1978.
[4] R. K. Mohanty, M. K. Jain, and K. George, "On the use of high order difference methods for the system of one space second order non-linear hyperbolic equations with variable coefficients," Journal of Computational and Applied Mathematics, vol. 72, no. 2, pp. 421-431, 1996.

[5] R. K. Mohanty and U. Arora, "A new discretization method of order four for the numerical solution of one-space dimensional second-order quasi-linear hyperbolic equation," International Journal of Mathematical Education in Science and Technology, vol. 33, no. 6, pp. 829-838, 2002.

[6] R. K. Mohanty, U. Arora, and M. K. Jain, "Fourth-order approximation for the three space dimensional certain mildly quasilinear hyperbolic equation," Numerical Methods for Partial Differential Equations, vol. 17, no. 3, pp. 277-289, 2001.

[7] R. K. Mohanty, U. Arora, and M. K. Jain, "Linear stability analysis and fourth-order approximations at first time level for the two space dimensional mildly quasi-linear hyperbolic equations," Numerical Methods for Partial Differential Equations, vol. 17, no. 6, pp. 607-618, 2001.

[8] M. Dehghan and A. Shokri, "A numerical method for solving the hyperbolic telegraph equation," Numerical Methods for Partial Differential Equations, vol. 24, no. 4, pp. 1080-1093, 2008.

[9] M. Dehghan and A. Ghesmati, "Solution of the second-order one-dimensional hyperbolic telegraph equation by using the dual reciprocity boundary integral equation (DRBIE) method," Engineering Analysis with Boundary Elements, vol. 34, no. 1, pp. 51-59, 2010.

[10] A. Saadatmandi and M. Dehghan, "Numerical solution of hyperbolic telegraph equation using the Chebyshev Tau method," Numerical Methods for Partial Differential Equations, vol. 26, no. 1, pp. 239-252, 2010.

[11] M. Dehghan and M. Lakestani, "The use of Chebyshev cardinal functions for solution of the second-order one-dimensional telegraph equation," Numerical Methods for Partial Differential Equations, vol. 25, no. 4, pp. 931-938, 2009.

[12] L.-B. Liu and H.-W. Liu, "Quartic spline methods for solving one-dimensional telegraphic equations," Applied Mathematics and Computation, vol. 216, no. 3, pp. 951-958, 2010.

[13] M. Esmaeilbeigi, M. M. Hosseini, and S. T. Mohyud-Din, "A new approach of the radial basis functions method for telegraph equations," International Journal of Physical Sciences, vol. 6, no. 6, pp. 1517-1527, 2011.

[14] V. R. Hosseini, W. Chen, and Z. Avazzadeh, "Numerical solution of fractional telegraph equation by using radial basis functions," Engineering Analysis with Boundary Elements, vol. 38, no. 12, pp. 31-39, 2014.

[15] A. Mohebbi, M. Abbaszadeh, and M. Dehghan, “The meshless method of radial basis functions for the numerical solution of time fractional telegraph equation," International Journal of Numerical Methods for Heat \& Fluid Flow, vol. 24, no. 8, pp. 1636-1659, 2014.

[16] W. T. Ma, B. W. Zhang, and H. L. Ma, "A meshless collocation approach with barycentric rational interpolation for twodimensional hyperbolic telegraph equation," Applied Mathematics and Computation, vol. 279, pp. 236-248, 2016.

[17] M. Dosti and A. Nazemi, "Quartic B-spline collocation method for solving one-dimensional hyperbolic telegraph equation," Journal of Information and Computing Science, vol. 7, no. 2, pp. 83-90, 2012.

[18] Sh. Sharifi and J. Rashidinia, "Numerical solution of hyperbolic telegraph equation by cubic B-spline collocation method," 
Applied Mathematics and Computation, vol. 281, pp. 28-38, 2016.

[19] R. C. Mittal and R. Bhatia, "Numerical solution of second order one dimensional hyperbolic telegraph equation by cubic Bspline collocation method," Applied Mathematics and Computation, vol. 220, pp. 496-506, 2013.

[20] N. Mollahasani, M. M. Moghadam, and K. Afrooz, "A new treatment based on hybrid functions to the solution of telegraph equations of fractional order," Applied Mathematical Modelling, vol. 40, no. 4, pp. 2804-2814, 2016.

[21] J. Rashidinia and M. Jokar, "Application of polynomial scaling functions for numerical solution of telegraph equation," Applicable Analysis, vol. 95, no. 1, pp. 105-123, 2016.

[22] K. T. Elgindy, "High-order numerical solution of second-order one-dimensional hyperbolic telegraph equation using a shifted Gegenbauer pseudospectral method," Numerical Methods for Partial Differential Equations, vol. 32, no. 1, pp. 307-349, 2016.

[23] R. Jiwari, S. Pandit, and R. C. Mittal, "A differential quadrature algorithm for the numerical solution of second order one dimensional hyperbolic telegraph equation," International Journal of Nonlinear Science, vol. 13, no. 3, pp. 259-266, 2012.

[24] R. K. Mohanty, "An unconditionally stable difference scheme for the one-space-dimensional linear hyperbolic equation," Applied Mathematics Letters, vol. 17, no. 1, pp. 101-105, 2004.

[25] R. K. Mohanty and M. K. Jain, "An unconditionally stable alternating direction implicit scheme for the two space dimensional linear hyperbolic equation," Numerical Methods for Partial Differential Equations, vol. 17, no. 6, pp. 684-688, 2001.

[26] R. K. Mohanty, M. K. Jain, and U. Arora, "An unconditionally stable ADI method for the linear hyperbolic equation in three space dimensions," International Journal of Computer Mathematics, vol. 79, no. 1, pp. 133-142, 2002.

[27] A. Borhanifar and R. Abazari, "An unconditionally stable parallel difference scheme for telegraph equation," Mathematical Problems in Engineering, vol. 2009, Article ID 969610, 17 pages, 2009.

[28] R. K. Mohanty, "New unconditionally stable difference schemes for the solution of multi-dimensional telegraphic equations," International Journal of Computer Mathematics, vol. 86, no. 12, pp. 2061-2071, 2009.

[29] F. Gao and C. M. Chi, "Unconditionally stable difference schemes for a one-space-dimensional linear hyperbolic equation," Applied Mathematics and Computation, vol. 187, no. 2, pp. 1272-1276, 2007.

[30] H.-W. Liu and L.-B. Liu, "An unconditionally stable spline difference scheme of $O\left(k^{2}+h^{4}\right)$ for solving the second-order 1D linear hyperbolic equation," Mathematical and Computer Modelling, vol. 49, no. 9-10, pp. 1985-1993, 2009.

[31] L.-B. Liu and H.-W. Liu, "Compact difference schemes for solving telegraphic equations with Neumann boundary conditions," Applied Mathematics and Computation, vol. 219, no. 19, pp. 10112-10121, 2013.

[32] S.-S. Xie, S.-C. Yi, and T. I. Kwon, "Fourth-order compact difference and alternating direction implicit schemes for telegraph equations," Computer Physics Communications, vol. 183, no. 3 , pp. 552-569, 2012.

[33] S. Chen, X. Jiang, F. Liu, and I. Turner, "High order unconditionally stable difference schemes for the Riesz space-fractional telegraph equation," Journal of Computational and Applied Mathematics, vol. 278, pp. 119-129, 2015.
[34] X. Q. Luo and Q. K. Du, "An unconditionally stable fourth-order method for telegraph equation based on Hermite interpolation," Applied Mathematics and Computation, vol. 219, no. 15, pp. 8237-8246, 2013.

[35] X. Luo and S. S.-T. Yau, "Hermite spectral method to 1-D forward Kolmogorov equation and its application to nonlinear filtering problems," IEEE Transactions on Automatic Control, vol. 58, no. 10, pp. 2495-2507, 2013.

[36] D. Funaro and O. Kavian, "Approximation of some diffusion evolution equations in unbounded domains by Hermite functions," Mathematics of Computation, vol. 57, no. 196, pp. 597-619, 1991.

[37] B.-Y. Guo, "Error estimation of Hermite spectral method for nonlinear partial differential equations," Mathematics of Computation, vol. 68, no. 227, pp. 1067-1078, 1999.

[38] B. Y. Guo and C. L. Xu, "Hermite pseudospectral method for nonlinear partial differential equations," Esaim Mathematical Modelling and Numerical Analysis, vol. 34, no. 4, pp. 859-872, 2000.

[39] H. Alıc1, "The Hermite pseudospectral method for the twodimensional Schrödinger equation with nonseparable potentials," Computers \& Mathematics with Applications, vol. 69, no. 6, pp. 466-476, 2015.

[40] H. Ma, W. Sun, and T. Tang, "Hermite spectral methods with a time-dependent scaling for parabolic equations in unbounded domains," SIAM Journal on Numerical Analysis, vol. 43, no. 1, pp. 58-75, 2005.

[41] H. Ma and T. Zhao, "A stabilized Hermite spectral method for second-order differential equations in unbounded domains," Numerical Methods for Partial Differential Equations, vol. 23, no. 5, pp. 968-983, 2007.

[42] X. Luo, S.-T. Yau, and S. S.-T. Yau, “Time-dependent HermiteGalerkin spectral method and its applications," Applied Mathematics and Computation, vol. 264, Article ID 21081, pp. 378-391, 2015.

[43] J. Shen and T. Tang, Spectral and High-Order Methods with Applications, Science Press, Beijing, China, 2006.

[44] Y.-S. Chung, T. K. Sarkar, B. H. Jung, and M. Salazar-Palma, "An unconditionally stable scheme for the finite-difference timedomain method," IEEE Transactions on Microwave Theory and Techniques, vol. 51, no. 3, pp. 697-704, 2003. 


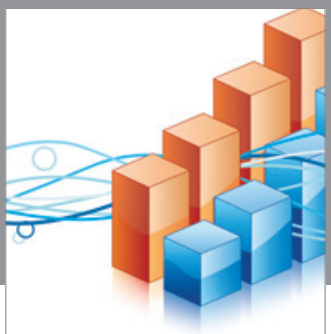

Advances in

Operations Research

vatem alat4

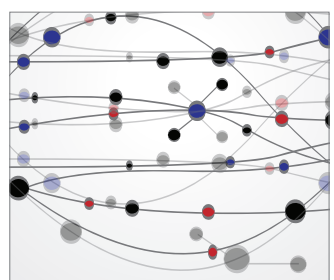

\section{The Scientific} World Journal
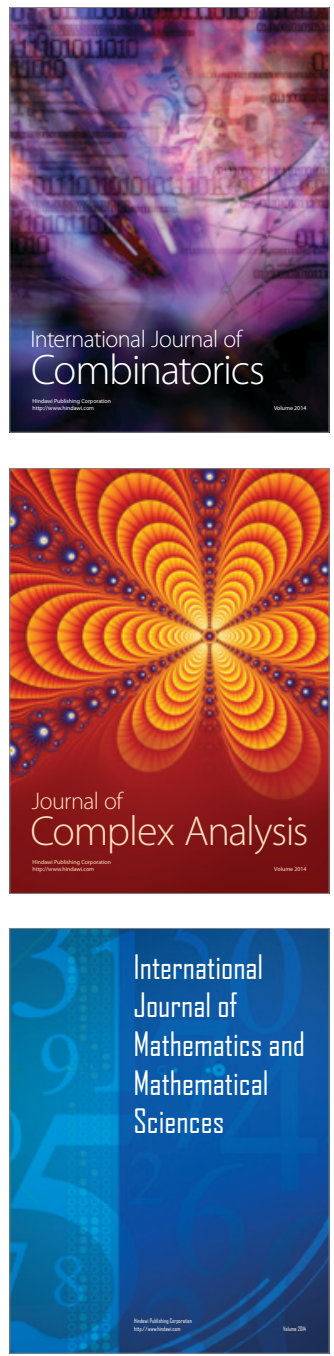
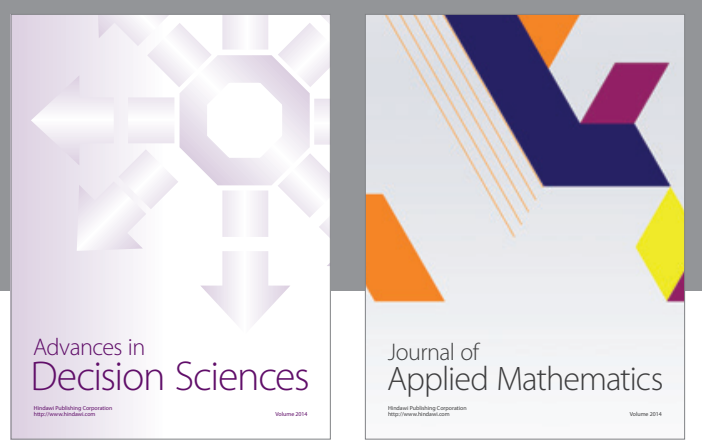

Algebra

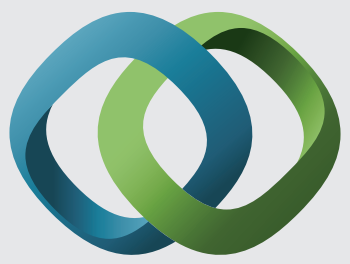

\section{Hindawi}

Submit your manuscripts at

http://www.hindawi.com
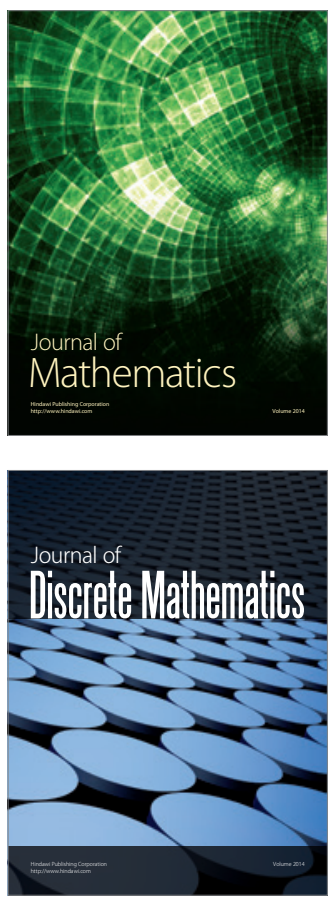

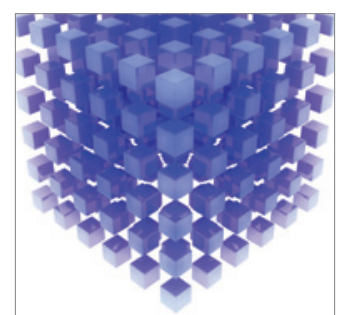

Mathematical Problems in Engineering
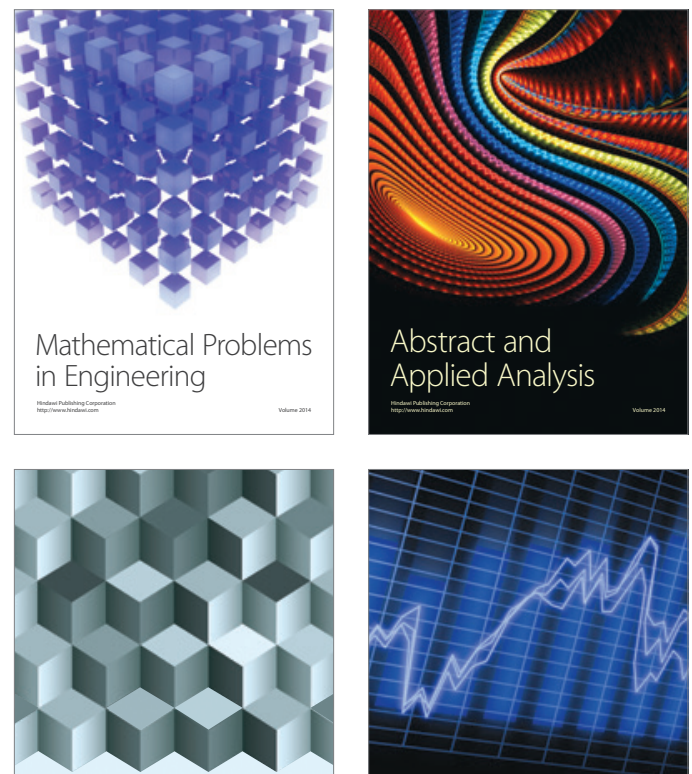

Journal of

Function Spaces

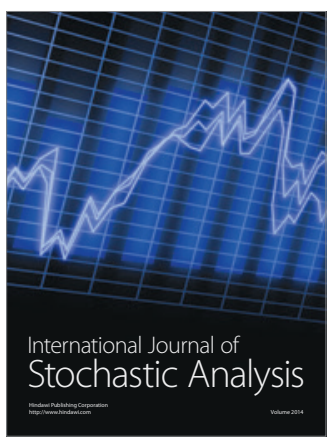

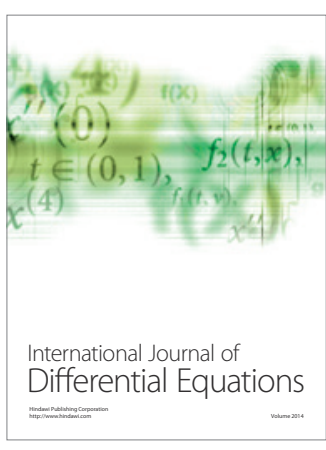
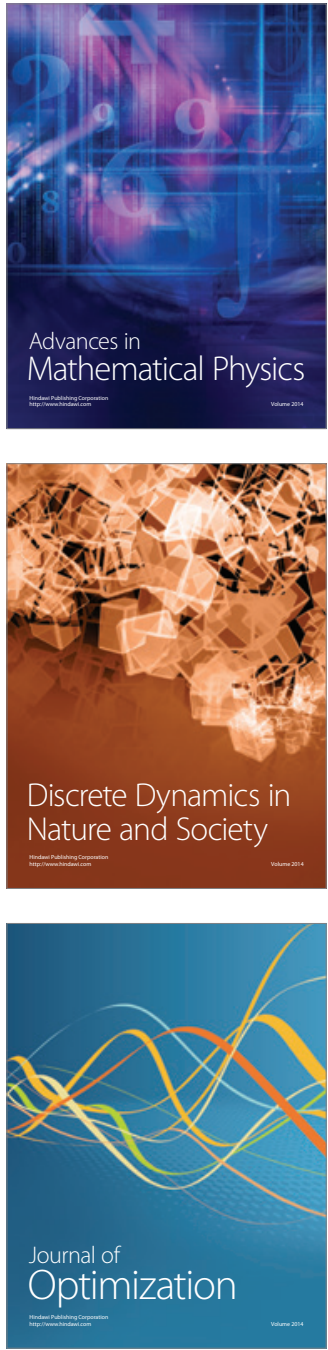